\title{
Fingerprint Based ATM Security by using ARM7
}

\author{
${ }^{1}$ D. Vinod kumar, ${ }^{2}$ Prof.M R K Murthy \\ ${ }^{1}$ Jayamukhi Institute of technology and science $\{$ MTech $\}$ Warangal, India \\ ${ }^{2}$ Jayamukhi Institute of technology and science $\{$ MTech\} Warangal,
}

\begin{abstract}
The purpose of this project is to increase the security that customer use the ATM machine. Once user's bank card is lost and the password is stolen, the criminal will draw all cash in the shortest time, which will bring enormous financial losses to customer, so to rectify this problem we are implementing this project. The chip of LPC2148 is used for the core of microprocessor in ARM7, furthermore, an improved enhancement algorithm of fingerprint image increase the security that customer use the ATM machine.
\end{abstract}

Keywords: ATM terminal; ARM7; fingerprint recognition, Image Enhancement, GSM MODEM.

\section{Introduction}

Here in this project we are going provide the at most security since it is taking the FINGER PRINTS as the authentication for our account. So whenever we want to access our account first we have to press the finger on the finger print scanner. Scanner is interfaced to the micro controller with the serial interfacing. The micro controller reads the data from the scanner. The micro controller allows those users, who are authorized to operate the account. If any unauthorized user tries to operate the account the micro controller switches on the security alarm. The total information about the account holders is stored in the EEPROM. Keypad is used to enter the password to operate the account or Locker. In present days, computer becomes a main part of human beings for storing information. This information is up to some extent is a secured one. For example the details of employees and students etc... The authority person may only change the details. For this protection we are going to provide a PASSWORD for the PCs. This is secure up to some extent only because there may be a chance of revealing the password or sometimes the authorized person may forgot the password. So we have to provide security for PCs with a unique and simple to remember identification. One of such identification is the FINGER PRINT. Fingerprint Scanner is a device for computer Security featuring superior performance, accuracy, durability based on unique NITGEN Fingerprint Biometric Technology. Fingerprint Scanner can be plugged into a computer separately with your mouse. Fingerprint Scanner is very safe and convenient device for security.

\section{The Characteristics Of The System Design}

The embedded ATM client authentication system is based on fingerprint recognition which is designed after analyzed existed ATM system. The LPC2148 chip is used as the core of these embedded system which is associated with the technologies of fingerprint recognition and current high speed network communication.

The primary functions are shown as follows:

- Fingerprint recognition: The masters' fingerprint information was used as the standards of identification. It must certify the feature of the human fingerprint before using ATM system.

- Remote authentication: System can compare current client's fingerprint information with remote fingerprint data server.

- Message alarming: different 4-digit code as a message to the mobile of the authorized customer without any noise, in order to access the Terminal.

- Two discriminate analysis methods: Besides the fingerprint recognition, the mode of password recognition can be also used for the system.

\section{Hardware Design and Software Design}

The design of entire system consisted of two part which are hardware and software. The hardware are designed by the rules of embedded system, and the steps of software consisted of three parts. The more details are shown as follows.

A. Hardware Design

The LPC2148 chip is used as the core of entire hardware. Furthermore, the modules of LCD, keyboard, alarm, fingerprint recognition are connected with the main chip (LPC2148).The EEPROM are also embodied in the system. There are some modules consisted of the system as follows

- LCD module: The $16 \mathrm{X} 2$ is used in this module as a LCD Display, it supported $5 \times 7$ matrix

- keyboard module: It can be used for inputting passwords. 
- Fingerprint recognition module: Nitgen Company's be used as a fingerprint recognition. It has a 100dpi resolution, anti-press, anti-static, anticorrosion.

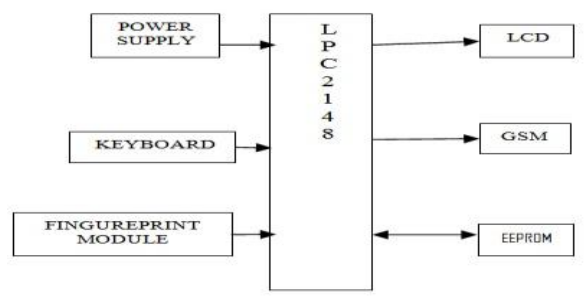

Figure I. The block diagram of hardware

B. Software design

The design was component of three parts included the design of main program flow chart, the initializing ones, and the algorithm of fingerprint recognition flow chart.

This system of software is implemented by the steps as follows: first of all, the Linux kernel and the File system are loaded into the main chip. The next, the system is initialized to implement specific task, such as checking ATM system, GSM communication and so on, and then each module reset for ready to run commands. Before using ATM terminal, the mobile number and fingerprint of the customer is required.

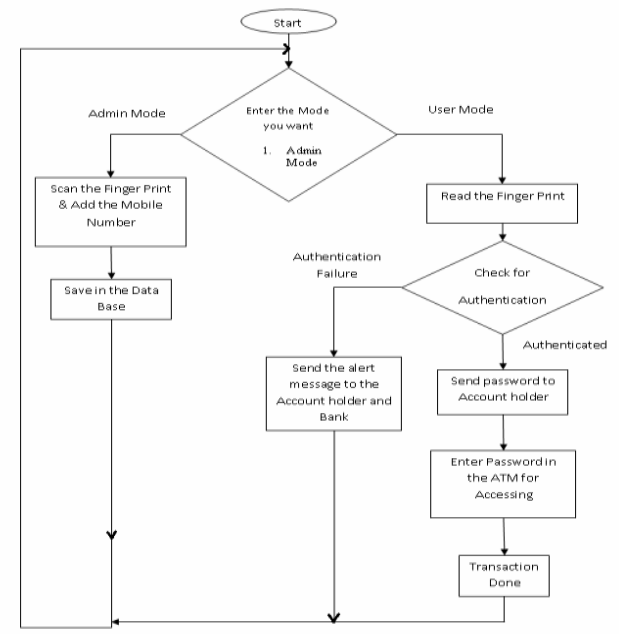

Figure 2. The overall flow chart of software

First the system is required the owner's fingerprint. If all the recognition is right, the system would send password to the Account holder and he will enter the same password in touch screen for accessing the ATM Terminal. If Authentication Failure then it send the alert message to the Account holder and Bank. The overall flow chart of software is shown in Figure 2.

In the process of inputting fingerprint, the AT77CI04B which is a linear sensor that captures fingerprint images by sweeping the finger over the sensing area, will used for acquiring the image of fingerprint. This product embed true hardware based 8-way navigation and click functions. The fingerprint information will be temporarily stored in SRAM and upload to the remote finger data server to compare through bank network. The result of process will be controlled by main chip(LPC 2148).

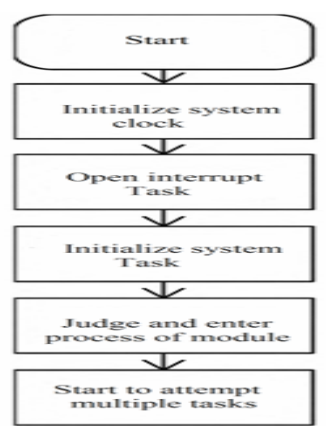

Figure3 The flow chart of fingerprint recognition 
The initializing process means that set the hardware and software and then start the multiple mission module, each module will be started according to the priority processes. At first, initialize the system clock, and execute the codes of open interrupt and the open interrupt task. Then, the system would judge and enter process of module. finally, the system would start to attempt multiple tasks. The initializing flow chart is shown in figure 3 .

C. The design of fingerprint recognition

Fingerprints are one of many forms of biometrics, used to identify individuals and verify their identity. This article touches on two major classes of algorithms and four sensor designs (optical, ultrasonic, passive capacitance, and active capacitance) The analysis of fingerprints for matching purposes generally requires the comparison of several features of the print pattern. These include patterns, which are aggregate characteristics of ridges, and minutia points, which are unique features found within the patterns It is also necessary to know the structure and properties of human skin in order to successfully employ some of the imaging technologies

The three basic patterns of fingerprint ridges are the arch, loop, and whorl:

- $\quad$ arch: The ridges enter from one side of the finger, rise in the center forming an arc, and then exit the other side of the finger.

- loop: The ridges enter from one side of a finger, form a curve, and then exit on that same side.

- $\quad$ whorl: Ridges form circularly around a central point on the finger.

The first step was the acquisition of fingerprint image by above device mentioned in the algorithm, and the results could be sent to the following process. secondly, pre-processing the images acquired. After obtain the fingerprint image, it must be pre-processing. Generally, pre-processing of one's is filtering, histogram computing, image enhancement and image binarization. Lastly, the characteristic value was extracted, and the results of the above measures would be compared with the information of owner's fingerprint in the database so as to verify whether the character is matched, and then the system returned the results matched or not.

\section{GSM}

Global System for Mobile Communication (GSM) is a set of ETSI standards specifying the infrastructure for a digital cellular service. The standard is used in approx. 85 countries in the world including such locations as Europe, Japan and Australia. GSM security issues such as theft of service, privacy, and legal interception continue to raise significant interest in the GSM community. The purpose of this portal is to raise awareness of these issues with GSM security.

The mobile communications has become one of the driving forces of the digital revolution. Every day, millions of people are making phone calls by pressing a few buttons. Little is known about how one person's voice reaches the other person's phone that is thousands of miles away. Even less is known about the security measures and protection behind the system. The complexity of the cell phone is increasing as people begin sending text messages and digital pictures to their friends and family. The cell phone is slowly turning into a handheld computer. All the features and advancements in cell phone technology require a backbone to support it. The system has to provide security and the capability for growth to accommodate future enhancements. General System for Mobile Communications, GSM, is one of the many solutions out there. GSM has been dubbed the "Wireless Revolution" and it doesn't take much to realize why GSM provides a secure and confidential method of communication.

\section{Results \& Conclusions}

The design of ATM terminal system based on finger print recognition took advantages of the stability and reliability of fingerprint characteristics, a new biological technology based on the image enhancement algorithm of Gabor and direction filter. Additional, the system also contains the original verifying methods which was inputting owner's password. The security features were The design of ATM terminal system based on finger print recognition took advantages of the stability and reliability of finger print characteristics, a new biological technology based on the image enhancement algorithm of Gabor and direction filter. Additional, the system also contains the original verifying methods which was inputting owner's password. The security features were

\section{REFERENCES}

[1] Lin Hong, Wan Yifei, Anil Jain. Fingerprint image enhancement: algorithm and performance evaluation[J]. IEEE Transactions on Pattern Analysis and Machine intelligence. 1998,20(8): 777-789.

[2] E Saatci, V Tavsanogh. Fingerprint image enhancement using CNN gabor-Cpe filter[C]. Proceedings of the 7th IEEE International Workshop on Cellular Neural Networks and their Applications 2002: 377-382.

[3] Gu J, Zhou J, Zhang D.A combination model for orientation field of fingerprints. Pattern Recognition 2004, $37: 543-553$.

[4] Cheng J, Tian J. Fingerprint enhancement with dyadic scale-space. Pattern Recognition Letters, 2004, 25(11): $1273-1284$.

[5] Chen H, Tian J. A fingerprint matching algorithm with registration pattern inspection. Journal of Software, 2005,16(6): 1046-105.

[6] Smits G FJordaan E M. Improved SVM Regression using Mixtures of Kernels [A]. Proceedings of the 2002 International Joint Conference on Neural Networks[C]. Hawaii: IEEE. 2002. 2785-2. 